\title{
Globally stable tracking and estimation for single-phase electrical signals with DC-offset rejection
}

\author{
Gilberto Pin ${ }^{\mathrm{a}}$, Boli Chen ${ }^{\mathrm{b}}$, Giuseppe Fedele ${ }^{\mathrm{c}}$, Thomas Parisini ${ }^{\mathrm{d}}$
}

\begin{abstract}
The present work introduces a new algorithm, named Global Quadrature PLL (GQPLL) for tracking a sinusoidal signal and for estimating its frequency and amplitude. The proposed technique derives from the well-known PLL architecture based on Quadrature Signal Generation (QSG), that is widely used for tracking the fundamental of single-phase electrical signals. The proposed algorithm improves the existent QSG-PLL solutions from two different perspectives. First, the cancellation of the DC-bias is embedded by construction. Moreover, a Lyapunov-based stability analysis guarantees the global convergence of the estimates for arbitrarily large adaptation gains, enabling fast adaptation transients. The provided simulations show that the algorithm is able to deal with sudden variations of the fundamental frequency and of the DC-bias magnitude.
\end{abstract}

\section{INTRODUCTION}

The electrical grid has undergone substantial changes during the last decade and further structural modifications are foreseen in the next years. The reason for these changes is twofold: the rise of distributed power generation systems and the integration of renewable energy sources subjected to natural variability. Such a trend to decentralization, paired to the increased unpredictability of these new power sources, greatly complicates the management of the electrical grid. As a consequence, all the power devices requiring grid synchronization face the challenge of adapting to time-varying grid-voltage conditions, both in amplitude and frequency. Nevertheless, this problem is magnified in small grids or in those parts of the network undergoing islanded operation due to faults or maintenance. All of these arguments have motivated the research for high-performance grid-synchronization algorithms with frequency-adaptive features, able to cope with rapidly changing conditions.

The most common algorithms developed for grid synchronization consist of modified Phase-Locked-Loops (PLL) provided of frequency adaptation. The recent work [1] offers a quite detailed overview of the prevalent architectures used in single-phase applications, focusing in particular on those methods using Quadrature Signal Generation (QSG). In the

\footnotetext{
a G. Pin is with the Global Connectivity \& Technology organization of Electrolux Italia S.p.A., Italy ( $g i l b e r t o p i n @ a l i c e . i t)$;

$\mathrm{b}$ B. Chen is with the Dept. of Electrical and Electronic Engineering, Imperial College London, UK (boli.chen10@imperial.ac.uk);

G. Fedele is with the Dept. of Informatics, Modeling, Electronics and System Engineering, University of Calabria, Italy (giuseppe. fedeledunical.it);

d T. Parisini is with the Dept. of Electrical and Electronic Engineering, Imperial College London, UK. He's also with the Dept. of Engineering and Architecture, Univ. of Trieste, Italy and with the KIOS Research and Innovation Centre of Excellence, University of Cyprus, Cyprus (t.parisini@gmail.com)
}

literature, QSG units are sometimes also referred to as Orthogonal Signal Generators (OSG). The Frequency LockedLoop (FLL) of [2], [3] based on a Second Order Generalized Integrator (SOGI) to implement the OSG, is for instance capable of tracking sinusoids with time-varying frequency and amplitude. Other algorithms taking origin from the very same idea of providing frequency-adaptive capability to a PLL can be found in [4] and [5]. The Enhanced PLL (EPLL) of [6], [7] is a successful design that has found extensive application to the estimation of power-grid signal parameters. Compared to a conventional PLL, its output signal is locked both in phase and in amplitude with the input. While the original EPLL is not robust to the presence of DC-bias, several further modifications have been proposed to overcome this limitation. The reader is referred to [8] for a modified EPLL based on delayed-signal cancellation that features the rejection of DC-bias. The problem of rejecting the DC-offset has been addressed also by [9] and [10].

The single-phase fundamental tracking problem tackled by the aforementioned algorithms can be boiled-down to the problem of estimating/tracking the following sinusoidal signal

$$
\begin{aligned}
& s(t)=A \sin \left(\vartheta(t)+\vartheta_{0}\right) \\
& \dot{\vartheta}(t)=\omega \\
& \vartheta(0)=0
\end{aligned}
$$

given the following biased/perturbed measurement

$$
y(t)=s(t)+c+\eta(t)
$$

where $c$ is an unknown scalar constant and where $\eta(t)$ is an unstructured perturbation term. The fundamental frequency $\omega$, the amplitude $A$ and the initial angle $\vartheta_{0}$ of the sinusoid are unknown. We assume that $\omega>\underline{\omega}$, with $\underline{\omega}>0$ a known lower bound on the angular frequency of the signal.

In the specific realm of power-electrical signal estimation, the unstructured perturbation term $\eta(t)$ contains the lumped contribution of unknown higher-order harmonics, inter-harmonics, measurement noise or quantization effects of the measurement front-end. When not dealt with explicitly by the algorithm (see for instance [11] and [12]) all these perturbations together may be considered as an exogenous bounded disturbance affecting the measured signal. This is indeed the typical setting of PLL-based methods aimed at tracking/estimating the fundamental. In this setting, providing a formal stability guarantee for the estimation algorithm is mandatory, at least with local properties.

Abstracting from the specific power-grid signal estimation problem, several solutions to the sinusoidal estimation 
problem outlined above have been conceived in the systemtheoretic and signal processing fields, and many results are given in terms of adaptive observers (see [13], [14], [15], [16], [17], [18], [19] among others), or nonlinear adaptive systems [20], [21], [22]. Notably, all of these methods formally guarantee the global stability of the estimator. Despite coming along with global stability guarantees, these methods are not widely used in the electrical-engineering field, mainly due to their complexity and the lack of an extensive, comparable sample of practical case-studies. On the contrary the modified-PLL methodologies listed above, tailored for power-electrical applications and supported by experimental results, represent the preferred solution in this field, even if only supported by local stability proofs. Indeed, the formal stability proofs of SOGI-OSG-FLL and of most QSG-PLLs are based either on small-signal analysis (local linearization of the dynamics), either on averaging. The latter approach invokes the use of unpractical limiting conditions for the adaptation gains. The same conclusion can be drawn for the EPLL scheme [6], whose proof is based on variational arguments. As a final result, the adaptation rate for the estimated frequency must be tuned accurately to guarantee the stability of these algorithms in all the operating conditions. Typically, boosting the adaptation gains improves the convergence rate when the initial guess on the frequency is close to the true one and for clean measurements, but it may cause instability for large initialization error or in presence of measurement perturbations. In other words, global convergence may get lost in practice.

In this work, a frequency-adaptive QSG-PLL is devised that admits a Lyapunov function which guarantees the global asymptotic stability of the adaptive system. The proposed algorithm is given the name Global Quadrature PLL (GQPLL), in order to underline the fact that the Lyapunov-based global stability proof certifies the global convergence of the estimator without invoking small-gain arguments. Instead, the adaptation gain can be made arbitrarily large in order to achieve a faster convergence rate, while preserving global stability properties. Moreover, the global stability property is guaranteed also in presence of DC-offset, which is accounted for natively by the proposed methodology.

For the sake of the further discussion, let us consider the equivalent trigonometric expression of the signal $y(t)$, where we neglect $\eta(t)$ for the purpose of algorithm design:

$$
y(t)=a \sin (\vartheta(t))+b \cos (\vartheta(t))+c,
$$

where $a, b \in \mathbb{R}$ are unknown parameters related to the amplitude by $A=\sqrt{a^{2}+b^{2}}$. Letting $\hat{\Omega}(t)$ be the estimate for the squared-frequency $\Omega:=\omega^{2}$, we aim at tracking $y(t)$ by an estimated signal $\hat{y}(t)$ having the following structure

$$
\begin{aligned}
& \hat{y}(t)=\hat{a}(t) \sin (\hat{\vartheta}(t))+\hat{b}(t) \cos (\hat{\vartheta}(t))+\hat{c}_{0}(t) \\
& \dot{\hat{\vartheta}}(t)=\sqrt{\hat{\Omega}(t)}, \hat{\Omega}(t) \geq \underline{\Omega}, \quad \forall t \geq 0
\end{aligned}
$$

where we have introduced the lower bound $\underline{\Omega}:=\underline{\omega}^{2}$ (known conservatively) and where $\hat{c}_{0}(t)$ is a DC-bias compensation term.
The tracking objective can be attained by designing adaptation laws for $\hat{a}(t), \hat{b}(t), \hat{c}_{0}(t)$ and $\hat{\Omega}(t)$ such that $\lim _{t \rightarrow+\infty} \hat{y}(t)-y(t)=0$ and all the internal signals remain bounded, with the estimated frequency constrained in the admissible set $\mathcal{A}:=\{\omega \in \mathbb{R}: \omega \geq \underline{\omega}\}$. For the sake of notational simplicity, in the sequel we will neglect the explicit dependence from time of the signals and of the timevarying estimated parameters.

\section{GQPLL EQUATIONS}

In this Section we provide the complete set of equations needed to implement the sinusoidal tracker/estimator. The reader can refer to Figure 1 for a block diagram representation of the GQPLL.

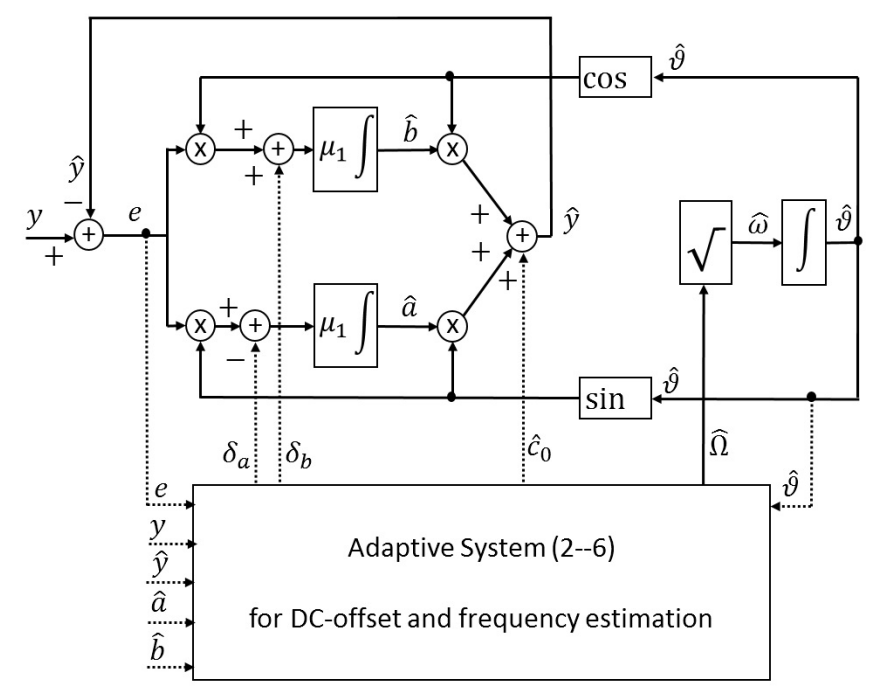

Fig. 1. Scheme of the GQPLL. The dashed lines denote the additional signal paths compared to a conventional QSG-PLL.

The equations for the online adaptation of the parameters $\hat{a}$ and $\hat{b}$ are given by the QSG-PLL-like multiplicative stage:

$$
\begin{aligned}
& \dot{\hat{a}}=\mu_{1} \sin (\hat{\vartheta}) e-\delta_{a} \\
& \dot{\hat{b}}=\mu_{1} \cos (\hat{\vartheta}) e+\delta_{b}
\end{aligned}
$$

with $\mu_{1}>0$ a constant adaptation gain (chosen by the designer to tune the convergence speed) and where the signals $\delta_{a}$ and $\delta_{b}$ are suitable nonlinear injections aimed at stabilizing the GQPLL. These signals are designed as

$\delta_{a}:=\sqrt{\hat{\Omega}} \cos (\hat{\vartheta})\left(\eta_{0} \hat{c}_{0}+\eta_{1}\left(\hat{c}_{1}+\sqrt{\hat{\Omega}}(\hat{b} \sin (\hat{\vartheta})-\hat{a} \cos (\hat{\vartheta}))\right)\right)$
$\delta_{b}:=\sqrt{\hat{\Omega}} \sin (\hat{\vartheta})\left(\eta_{0} \hat{c}_{0}+\eta_{1}\left(\hat{c}_{1}+\sqrt{\hat{\Omega}}(\hat{b} \sin (\hat{\vartheta})-\hat{a} \cos (\hat{\vartheta}))\right)\right)$

with $\hat{c}_{0}$ and $\hat{c}_{1}$ obtained through the second-order nonlinear filter:

$$
\begin{aligned}
& \dot{\hat{c}}_{0}=\hat{c}_{1}+\sqrt{\hat{\Omega}}(\hat{b} \sin (\hat{\vartheta})-\hat{a} \cos (\hat{\vartheta})) \\
& \dot{\hat{c}}_{1}=\left(\mu_{0}-\hat{\Omega}\right) e-\hat{\Omega} \hat{y}+\hat{K}
\end{aligned}
$$

where $\mu_{0}>0, \eta_{0}<0$ and $\eta_{1}<0$ are design constants that can be chosen arbitrarily. The stability analysis will show later the effect of these parameters on the convergence speed. 
Finally, the estimates for the squared-frequency $\hat{\Omega}$ and for the auxiliary parameter $\hat{K}$ are given by

$$
\begin{gathered}
\hat{\Omega}:=\max \left(\underline{\Omega},-\frac{k_{1}}{2} y^{2}+\hat{\Theta}_{\Omega}\right) \\
\hat{K}:=k_{1} y+\hat{\Theta}_{K}
\end{gathered}
$$

where

$$
\dot{\hat{\Theta}}_{\Omega}=k_{1} y \dot{\hat{y}}-k_{0} y e .
$$

and

$$
\dot{\hat{\Theta}}_{K}=-k_{1} \dot{\hat{y}}+k_{0} e .
$$

Note that the signal $\dot{\hat{y}}$ needed to implement the above adaptation laws can be obtained from available quantities without direct differentiation:

$$
\begin{aligned}
\dot{\hat{y}}= & \dot{\hat{a}} \sin (\hat{\vartheta})+\dot{\hat{b}} \cos (\hat{\vartheta})+\sqrt{\hat{\Omega}}(\hat{a} \cos (\hat{\vartheta})-\hat{b} \sin (\hat{\vartheta}))+\dot{\hat{c}}_{0} \\
= & \sin (\hat{\vartheta})\left(\mu_{1} \sin (\hat{\vartheta}) e-\delta_{a}\right)+\cos (\hat{\vartheta})\left(\mu_{1} \cos (\hat{\vartheta}) e+\delta_{b}\right) \\
& +\hat{c}_{1}+\sqrt{\hat{\Omega}}(\hat{b} \sin (\hat{\vartheta})-\hat{a} \cos (\hat{\vartheta})) \\
& +\sqrt{\hat{\Omega}}(\hat{a} \cos (\hat{\vartheta})-\hat{b} \sin (\hat{\vartheta})) .
\end{aligned}
$$

The expression for the estimated squared-frequency (3) is provided of a one-sided saturation that keeps the estimate within the admissible set of strict-positive values: $\hat{\omega}=\sqrt{\hat{\Omega}} \in \mathcal{A}$. All in all, the GQPLL has a total dynamical order of 7 with states $\hat{\Theta}_{k}, \hat{\Theta}_{\Omega}, \hat{c}_{0}, \hat{c}_{1}, \hat{a}, \hat{b}, \hat{\vartheta}$.

\section{Stability ANALysis}

In absence of saturation, the expression for the squaredfrequency (3) yields to the following parameter derivative

$$
\dot{\hat{\Omega}}=-k_{1} y \dot{y}+k_{1} y \dot{\hat{y}}-k_{0} y e=-k_{1} y \dot{e}-k_{0} y e
$$

whilst it holds that $\dot{\hat{\Omega}}=0$ when the saturation is active. Note that the above expression for $\dot{\hat{\Omega}}$, unlike the equations given in the previous section, is not directly implementable due to the fact that $\dot{e}$ is not available, but will be useful to study the closed-loop stability of the adaptive system. The frequency-error dynamics accounting for the saturation can be formally expressed by the following o.d.e. with possibly discontinuous right-hand side:

$$
\dot{\tilde{\Omega}}= \begin{cases}k_{1} y \dot{e}+k_{0} y e & ,(\hat{\Omega}>\underline{\Omega}) \vee\left(\frac{-k_{1} y \dot{e}-k_{0} y e}{2 \sqrt{\hat{\Omega}}}>0\right) \\ 0 & ,(\hat{\Omega}==\underline{\Omega}) \wedge\left(\frac{-k_{1} y \dot{e}-k_{0} y e}{2 \sqrt{\hat{\Omega}}} \leq 0\right)\end{cases}
$$

whose solutions are intended in the sense of Filippov, [23].

Now, let us take the time-derivative of the error $e:=y-\hat{y}$ :

$$
\begin{aligned}
\dot{e} & =\omega a \cos (\vartheta)-\omega b \sin (\vartheta)-\dot{\hat{a}} \sin (\hat{\vartheta})-\hat{a} \sqrt{\hat{\Omega}} \cos (\hat{\vartheta}) \\
& -\dot{\hat{b}} \cos (\hat{\vartheta})+\hat{b} \sqrt{\hat{\Omega}} \sin (\hat{\vartheta})-\dot{\hat{c}}_{0}
\end{aligned}
$$

Substituting the expression for $\dot{\hat{c}}_{0}$ then (8) simplifies into

$$
\dot{e}=\omega a \cos (\vartheta)-\omega b \sin (\vartheta)-\dot{\hat{a}} \sin (\hat{\vartheta})-\dot{\hat{b}} \cos (\hat{\vartheta})-\hat{c}_{1}
$$

Substituting the expressions for $\delta_{a}$ and $\delta_{b}$ in (1) we get

$$
\begin{aligned}
& \dot{\hat{a}}=\mu_{1} \sin (\hat{\vartheta}) e-\sqrt{\hat{\Omega}} \cos (\hat{\vartheta})\left(\eta_{0} \hat{c}_{0}+\eta_{1} \dot{\hat{c}}_{0}\right) \\
& \dot{\hat{b}}=\mu_{1} \cos (\hat{\vartheta}) e+\sqrt{\hat{\Omega}} \sin (\hat{\vartheta})\left(\eta_{0} \hat{c}_{0}+\eta_{1} \dot{\hat{c}}_{0}\right) .
\end{aligned}
$$

Substituting the latter expressions into (9) we get the intermediate result

$$
\begin{aligned}
\dot{e}= & \omega a \cos (\vartheta)-\omega b \sin (\vartheta)-\hat{c}_{1} \\
& -\mu_{1} \sin (\vartheta)^{2} e+\sin (\vartheta) \cos (\hat{\vartheta}) \sqrt{\hat{\Omega}}\left(\eta_{0} \hat{c}_{0}+\eta_{1} \dot{\hat{c}}_{0}\right) \\
& \quad-\mu_{1} \cos (\hat{\vartheta})^{2} e-\cos (\hat{\vartheta}) \sin (\vartheta) \sqrt{\hat{\Omega}}\left(\eta_{0} \hat{c}_{0}+\eta_{1} \dot{\hat{c}}_{0}\right) \\
= & \omega a \cos (\vartheta)-\omega b \sin (\vartheta)-\hat{c}_{1} \\
& -\mu_{1} \sin (\vartheta)^{2} e-\mu_{1} \cos (\hat{\vartheta})^{2} e \\
= & \omega a \cos (\vartheta)-\omega b \sin (\vartheta)-\mu_{1} e-\hat{c}_{1}
\end{aligned}
$$

The second time-derivative of the error yields to:

$$
\ddot{e}=-\omega^{2}(y-c)-\mu_{1} \dot{e}-\dot{\hat{c}}_{1}
$$

Now, substituting the expression for $\dot{\hat{c}}_{1}$ into (10) we obtain

$$
\begin{aligned}
\ddot{e} & =-\Omega(y-c)-\mu_{1} \dot{e}+\hat{\Omega} e-\mu_{0} e+\hat{\Omega} \hat{y}-\hat{K} \\
& =-\Omega y+\Omega c-\mu_{1} \dot{e}+\hat{\Omega} y-\mu_{0} e-\hat{K} \\
& =-(\Omega-\hat{\Omega}) y+(K-\hat{K})-\mu_{1} \dot{e}-\mu_{0} e .
\end{aligned}
$$

where we have posed $K:=\Omega c$. Now, let us define $\tilde{K}:=K-\hat{K}$. The provided implementable expression (4) for the parameter $\hat{K}$ implies that $\dot{\tilde{K}}$ can be written as:

$$
\dot{\tilde{K}}=-\dot{\hat{K}}=-k_{1} \dot{y}+k_{1} \dot{\hat{y}}-k_{0} e=-k_{1} \dot{e}-k_{0} e
$$

As in the case of (7), the above derivative is not directly implementable, due to the unavailability of $\dot{e}$, but will be useful to study the closed-loop stability.

Defining the parameter vector $\theta:=\left[\begin{array}{ll}\Omega & K\end{array}\right]^{\top}$ and denoting by $\hat{\theta}:=\left[\begin{array}{ll}\hat{\Omega} & \hat{K}\end{array}\right]^{\top}$ its estimate and by $\xi=\left[\begin{array}{ll}-y & 1\end{array}\right]^{\top}$ the vector of regressors, then we obtain the expression:

$$
\ddot{e}=\xi^{\top}(\theta-\hat{\theta})-\mu_{1} \dot{e}-\mu_{0} e .
$$

Finally, defining the parameter error vector $\tilde{\theta}:=\theta-\hat{\theta}$, the tracking-error dynamics (13) can be re-arranged as

$$
\ddot{e}=\xi^{\top} \tilde{\theta}-\mu_{1} \dot{e}-\mu_{0} e .
$$

This differential equation is one of the ingredients needed to characterize the convergence of the tracking-error.

Studying the stability of equation (14) alone is not sufficient, however, to characterize the stability of the overall adaptive system, which also consists of the squaredfrequency adaptation and of the nonlinear filter (2) generating the signals $\hat{c}_{0}$ and $\hat{c}_{1}$ that are needed to create the auxiliary injections $\delta_{a}$ and $\delta_{b}$. In this connection, let us take the second derivative of $\hat{c}_{0}$

$$
\begin{aligned}
\ddot{\hat{c}}_{0}= & \hat{\Omega}\left(\eta_{0} \hat{c}_{0}+\eta_{1} \dot{\hat{c}}_{0}\right)+\left(\mu_{0}-\hat{\Omega}\right) e-\hat{\Omega} \hat{c}_{0}+\hat{K} \\
& +\frac{\dot{\hat{\Omega}}}{2 \sqrt{\hat{\Omega}}}(\hat{b} \sin (\hat{\vartheta})-\hat{a} \cos (\hat{\vartheta})) \\
= & \hat{\Omega}\left(\left(\eta_{0}-1\right) \hat{c}_{0}+\eta_{1} \dot{\hat{c}}_{0}\right)+v
\end{aligned}
$$




$$
v:=\left(\mu_{0}-\hat{\Omega}\right) e+\hat{K}- \begin{cases}(\hat{b} \sin (\hat{\vartheta})-\hat{a} \cos (\hat{\vartheta})) \frac{k_{1} y \dot{e}+k_{0} y e}{2 \sqrt{\hat{\Omega}}} & ,(\hat{\Omega}>\underline{\Omega}) \vee\left(\frac{-k_{1} y \dot{e}-k_{0} y e}{2 \sqrt{\hat{\Omega}}}>0\right) \\ 0 & ,(\hat{\Omega}==\underline{\Omega}) \wedge\left(\frac{-k_{1} y \dot{e}-k_{0} y e}{2 \sqrt{\hat{\Omega}}} \leq 0\right)\end{cases}
$$

where the signal $v$, defined in (15), can be seen as an external input to the above system, depending on the tracking-error and on the parameters. The stability analysis will be performed in two steps, considering that the overall adaptive system can be viewed as the forward connection of:

- A) a system consisting of the parameter-error dynamics (7), (12) plus the error system (14), which is then cascaded to the system at point $B$ );

- $B$ ) a non-autonomous dynamical system corresponding to the error-driven auxiliary dynamics (16).

Step $A$ ): Consider now the following candidate Lyapunov function for the system (7),(12) and (14) (recall that $\tilde{\theta}$ incorporates both the squared-frequency error $\tilde{\Omega}$ and $\tilde{K}$ ):

$$
V=\frac{1}{2}\left(\bar{e}^{\top} P \bar{e}+\tilde{\theta}^{\top} \tilde{\theta}\right) .
$$

with $\bar{e}^{\top}:=\left[\begin{array}{ll}e & \dot{e}\end{array}\right]^{\top}$ and

$$
P:=\left[\begin{array}{ll}
p_{11} & p_{12} \\
p_{12} & p_{22}
\end{array}\right]
$$

where $p_{11}, p_{12}, p_{22}$ must be chosen such that $P$ is positive definite. For the sake of the further discussion let us recast the candidate Lyapunov function in the following scalar form

$$
V=p_{11} e^{2}+p_{22} \dot{e}^{2}+2 p_{12} e \dot{e}+\tilde{\Omega}^{2}+\tilde{K}^{2} .
$$

We will provide a tuning criteria for the parameters of the adaptive system for which will exist a $P>0$ such that $V$ satisfies the assumptions of the Lyapunov-LaSalle (L-LS) stability theorem.

First, let us now make some comments on the saturated parameter adaptation law. Given the convexity of the admissible domain for the estimated squared-frequency, the saturation will prevent the increase of the quadratic-error term $\tilde{\Omega}^{2}$ in the candidate Lyapunov function whenever the unconstrained derivative of the squared-frequency points outside the admissible domain. Thus for the sake of proving the negative-definiteness of the derivative of $V$ along the system's trajectory, we can take the conservative route of considering only the unsaturated case.

Considering that $\dot{\tilde{\theta}}=-\dot{\hat{\theta}}$, the derivative of $V$ along the system's trajectory yields:

$$
\begin{aligned}
\dot{V} \leq & p_{11} e \dot{e}+p_{22} \dot{e} \ddot{e}+p_{12} e \ddot{e}+p_{12} \dot{e}^{2}-\tilde{\Omega} \dot{\hat{\Omega}}-\tilde{K} \dot{\hat{K}} \\
= & p_{11} e \dot{e}-p_{22} \tilde{\Omega} y \dot{e}+p_{22} \tilde{K} \dot{e}-p_{22} \mu_{1} \dot{e}^{2}-p_{22} \mu_{0} e \dot{e}+ \\
& -p_{12} \tilde{\Omega} y e+p_{12} \tilde{K} e-p_{12} \mu_{1} e \dot{e}-p_{12} \mu_{0} e^{2}+p_{12} \dot{e}^{2} \\
& +k_{1} \tilde{\Omega} y \dot{e}+k_{0} \tilde{\Omega} y e-k_{1} \tilde{K} \dot{e}-k_{0} \tilde{K} e \\
= & -p_{12} \mu_{0} e^{2}-\left(p_{22} \mu_{1}-p_{12}\right) \dot{e}^{2} \\
& +\left(p_{11}-p_{12} \mu_{1}-p_{22} \mu_{0}\right) e \dot{e} \\
& +\left(k_{0}-p_{12}\right) \tilde{\Omega} y e+\left(k_{1}-p_{22}\right) \tilde{\Omega} y \dot{e} \\
& -\left(k_{0}-p_{12}\right) \tilde{K} e-\left(k_{1}-p_{22}\right) \tilde{K} \dot{e}
\end{aligned}
$$

To make $\dot{V}$ negative definite with respect to the error-state, one may select the constant parameters $\mu_{0}>0, \mu_{1}>0$ and $k_{0}>0$ arbitrarily (i.e., there are no small-gain restrictions on the adaptation parameters). Then pick $p_{12}=k_{0}, p_{22}=$ $\frac{p_{12}+\bar{\mu}_{1}}{\mu_{1}}$, for some arbitrary

$$
\bar{\mu}_{1}>\frac{k_{0}^{2}-p_{12}^{2}}{p_{12}}=0
$$

and set $k_{1}=p_{22}$ and $p_{11}=p_{12} \mu_{1}+p_{22} \mu_{0}$. Then we get

$$
\begin{aligned}
\operatorname{det}(P) & =p_{22} p_{11}-p_{12}^{2}=\frac{p_{12}+\bar{\mu}_{1}}{\mu_{1}}\left(p_{12} \mu_{1}+p_{22} \mu_{0}\right)-k_{0}^{2} \\
& >p_{12}^{2}+\bar{\mu}_{1} p_{12}-k_{0}^{2}>0
\end{aligned}
$$

which ensures that $V$ is positive-definite. Moreover, with the above choice for the elements of $P$ we have that $\dot{V}$ is negative-definite in the error-state:

$$
\dot{V} \leq-p_{12} \mu_{0} e^{2}-\bar{\mu}_{1} \dot{e}^{2}
$$

We can conclude that $V$ is an L-LS function (positive definite radially unbounded with semi-negative-definite derivative) for the system (7),(14). Following standard arguments in adaptive control (Barbalat's lemma + La Salle's invariance principle, see [24] and [25]), it can be proven that $\tilde{\theta}$ converges to zero in case of conventional persistency of excitation conditions, which is always the case for a sinusoid of nonzero amplitude.

Step $B$ ): It remains to prove that that the auxiliary signals $\delta_{a}, \delta_{b}$ and $\hat{c}_{0}$ injected into the GQPLL to stabilize the dynamics remain bounded. Having already proven the boundedness of $\hat{\Omega}$, $\hat{a}$ and $\hat{b}$ at Step A), by considering the expressions for $\delta_{a}, \delta_{b}$ we can conclude that the proof can be completed by proving that $\lim _{t \rightarrow \infty}\left|\hat{c}_{0}\right|<+\infty$ and $\lim _{t \rightarrow \infty}\left|\hat{c}_{1}\right|<+\infty$. It holds that (16), is a stable linear system $\left(\eta_{0}<0\right.$ and $\eta_{1}<0$ by design) which is therefore ISS with respect to $v$. We proceed by showing that $v$ is bounded. Since the error $e \rightarrow 0$ and $y$ is bounded, then the output $\hat{y}$ of the estimator is bounded, too, which implies that also $\hat{a}$ and $\hat{b}$ are bounded. Moreover, the boundedness of $\tilde{K}$ descends from the boundedness of $\tilde{\theta}$. This fact in turn implies that $v$ is bounded. Since we have proved that the error-state $\left[\begin{array}{ll}e & \dot{e}\end{array}\right]^{\top} \rightarrow 0$ exponentially, the ISS of the cascaded system (16) implies that also $\hat{c}_{0}$ is bounded. The boundedness of $\hat{c}_{1}$ can be proven by invoking the same arguments.

In view of the above analysis, it turns out that the adaptation gains can be chosen arbitrarily large, enabling faster convergence while preserving global stability properties. The certifiable global stability of the GQPLL out of any smallgain assumption represents a key benefit over the existent QSG-PLL schemes. 


\section{Simulation Results: Grid Voltage Tracking}

The behavior of the proposed GQPLL is analyzed in this Section through some insightful numerical simulations, considering the estimation of grid-voltage parameters in both nominal and perturbed scenarios. For benchmarking purposes, the GQPLL is compared with the following algorithms: the EPLL of Kharimi-Garthemani [6], which is one of the most used architectures in the Electrical Engineering framework for the extraction of grid-voltage parameters, and the Adaptive Observer ( $\mathrm{AO}$ ) developed in [26], which is often taken as a baseline for comparison by the system-theoretic literature.

The following sinusoidal signal subjected to DC-offset is used to emulate a grid-voltage signal with rated $50 \mathrm{~Hz}$ frequency and subjected to sudden frequency-variations:

$$
y(t)=320 \sin (2 \pi f t)+c
$$

where

$$
f=\left\{\begin{array}{ll}
52.5 \quad 0 \leq t<0.4, \\
47.5, \quad t \geq 0.4,
\end{array} \quad, \quad c= \begin{cases}10, & 0 \leq t<1 \\
15, & t \geq 1\end{cases}\right.
$$

The parameters of the proposed GQPLL are chosen as $\mu_{0}=$ $5 e 4, \mu_{1}=200, k_{0}=5 e 5, k_{1}=2 e 4, \eta_{0}=-80, \eta_{1}=-15$. For the sake of comparison, all the methods are initialized with the same guess $50 \mathrm{~Hz}$ for the frequency. Moreover, both the EPLL and the AO are tuned so that their frequency estimates exhibit the same convergence speed of the GQPLL in the noise-free scenario (see Fig. 2). As shown in Fig. 2, the frequency change at $t=0.4 \mathrm{~s}$ can be captured by all the methods under concern with comparable response speed, but the GQPLL shows fewer oscillations. A remarkable feature of the proposed algorithm can be appreciated at time $t=1 \mathrm{~s}$, when the DC-bias term is suddenly changed: while the GQPLL estimate appears insensitive to this variation, instead the AO and the EPLL exhibit some oscillations. The reconstructed sinusoid of each method is depicted in Fig. 3. As it can be seen, despite the similar accuracy in steady state, the EPLL shows the best transient performance. Compared to the EPLL, the transient response of the GQPLL is slightly slower, while the AO requires much longer response time to address a parameter change than the other two algorithms.

Next, we examine the robustness of the three approaches in facing unstructured perturbations, assuming the measurement $y(t)$ is corrupted by a random noise uniformly distributed in the interval $[-10,10]$. It is found in Figs. 2 and 3 that the estimates of the proposed method remain accurate due to its higher noise immunity. The EPLL is also capable to extract the sine wave from the noisy measurement with satisfactory accuracy and slightly faster convergence speed than the GQPLL at the very beginning, however, its frequency estimates are susceptible to the additive noise. Although the frequency term is successfully tracked by the AO throughout the simulation, the presence of the noise causes distortions on the estimated synchronizing signal component. We can conclude that the EPLL should be preferred for tracking the sinusoidal grid-signal, while the GQPLL performs better

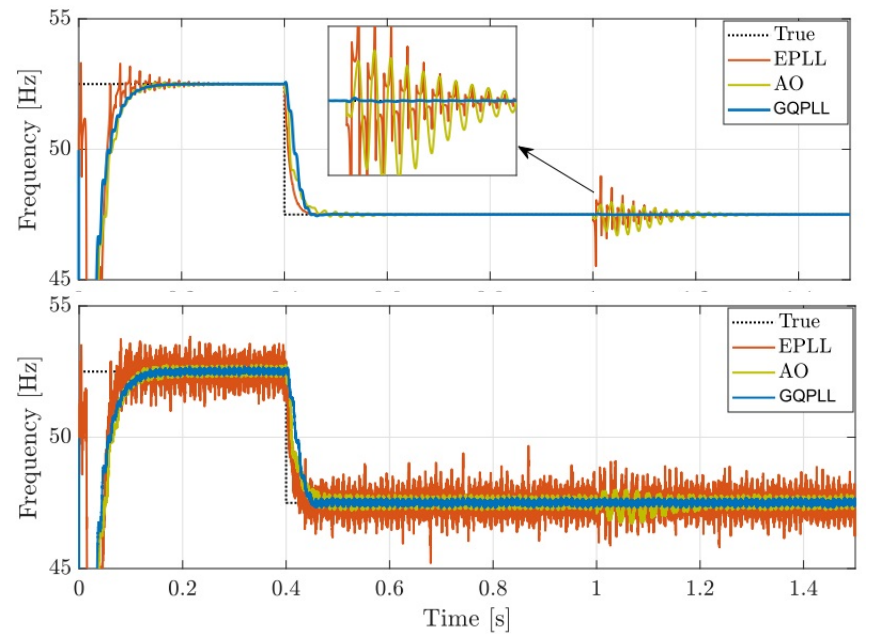

Fig. 2. Time behavior of the frequency estimates provided by the EPLL [7], the AO [26] and the GQPLL in the noise-free (top) and noisy (bottom) scenarios.

in terms of frequency-estimation, due to its superior DCrejection performance and noise immunity.

\section{CONCLUSION}

The paper proposes an estimation and tracking algorithm for DC-biased sinusoidal signals, named GQPLL, that brings together the architecture of QSG-PLL methods with the stability certificates of adaptive observers. The algorithm features the complete rejection of DC-bias and the ability to track frequency variations. The global asymptotic stability of the adaptive system is proven by Lyapunov analysis.

Further research will be devoted to characterize the behavior of the estimator in presence of harmonics and interharmonics, and to extend the methodology to three-phase systems with possible unbalance. Experimental activities will be also performed to evaluate the performance of the method in a realistic scenario.

\section{REFERENCES}

[1] S. Golestan, J. M. Guerrero, and J. C. Vasquez, "Single-phase PLLs: A review of recent advances," IEEE Trans on Power Electronics, vol. 32, no. 12, pp. 9013-9030, Dec 2017.

[2] G. Fedele and A. Ferrise, "A frequency-locked-loop filter for biased multi-sinusoidal estimation," IEEE Trans on Signal Processing, vol. 62, no. 5, pp. 1125-1134, March 2014.

[3] G. Fedele, A. Ferrise, and G. D'Aquila, "A global frequency estimator based on a frequency-locked-loop filter," in 2016 American Control Conference (ACC), July 2016, pp. 7001-7006.

[4] I. Carugati, P. Donato, S. Maestri, D. Carrica, and M. Benedetti, "Frequency adaptive PLL for polluted single-phase grids," IEEE Trans on Power Electronics, vol. 27, no. 5, pp. 2396-2404, May 2012.

[5] G. Pin, "A direct approach for the frequency-adaptive feedforward cancellation of harmonic disturbances," IEEE Trans on Signal Processing, vol. 58, no. 7, pp. 3523-3530, July 2010.

[6] M. Karimi-Ghartemani and M. R. Iravani, "A nonlinear adaptive filter for online signal analysis in power systems: applications," IEEE Trans on Power Delivery, vol. 17, no. 2, pp. 617-622, 2002.

[7] M. Karimi-Ghartemani, S. A. Khajehoddin, P. K. Jain, A. Bakhshai, and M. Mojiri, "Addressing DC component in PLL and notch filter algorithms," IEEE Trans on Power Electronics, vol. 27, no. 1, pp. 78-86, 2012. 

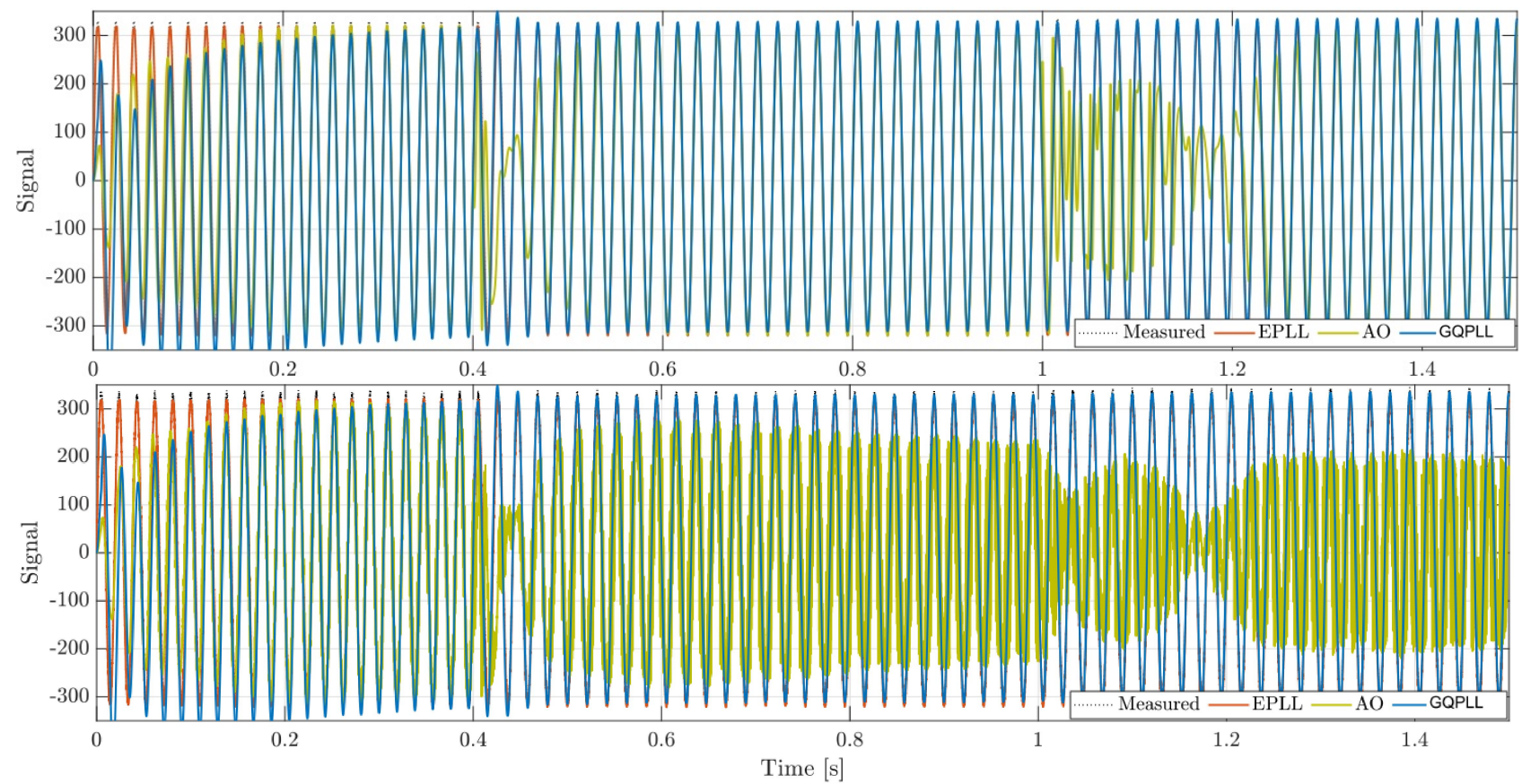

Fig. 3. Time behavior of the reconstructed sinusoidal voltage signal provided by the EPLL [7], the AO [26] and the GQPLL in the noise-free (top) and noisy (bottom) scenarios.

[8] F. Wu, D. Sun, L. Zhang, and J. Duan, "Influence of plugging DC offset estimation integrator in single-phase ePLL and alternative scheme to eliminate effect of input DC offset and harmonics," IEEE Trans on Industrial Electronics, vol. 62, no. 8, pp. 4823-4831, Aug 2015.

[9] S. Hwang, L. Liu, H. Li, and J. Kim, "DC offset error compensation for synchronous reference frame PLL in single-phase grid-connected converters," IEEE Trans on Power Electronics, vol. 27, no. 8, pp. 3467-3471, Aug 2012.

[10] S. Golestan, M. Monfared, F. D. Freijedo, and J. M. Guerrero, "Design and tuning of a modified power-based PLL for single-phase grid-connected power conditioning systems," IEEE Trans on Power Electronics, vol. 27, no. 8, pp. 3639-3650, Aug 2012.

[11] B. Chen, G. Pin, W. M. Ng, T. Parisini, and S. Y. R. Hui, "A fast-convergent modulation integral observer for online detection of the fundamental and harmonics in grid-connected power electronics systems," IEEE Trans on Power Electronics, vol. 32, no. 4, pp. 25962607, April 2017.

[12] B. Chen, G. Pin, W. M. Ng, P. Li, T. Parisini, and S. Y. R. Hui, "Online detection of fundamental and interharmonics in ac mains for parallel operation of multiple grid-connected power converters," IEEE Trans on Power Electronics, vol. PP, no. 99, pp. 1-1, 2018.

[13] M. Hou, "Estimation of sinusoidal frequencies and amplitudes using adaptive identifier and observer," IEEE Trans on Automatic Control, vol. 52, no. 3, pp. 493-499, March 2007.

[14] A. A. Bobtsov, N. A. Nikolaev, O. V. Slita, A. S. Borgul, and S. V. Aranovskiy, "The new algorithm of sinusoidal signal frequency estimation," IFAC Proceedings Volumes, vol. 46, no. 11, pp. 182 - 186, 2013, 11th IFAC Workshop on Adaptation and Learning in Control and Signal Processing.

[15] S. Aranovskiy, A. Bobtsov, A. Kremlev, N. Nikolaev, and O. Slita, "Identification of frequency of biased harmonic signal," European J. of Control, vol. 16, no. 2, pp. 129 - 139, 2010.

[16] B. Chen, G. Pin, W. M. Ng, C. K. Lee, S. Y. R. Hui, and T. Parisini, "An adaptive observer-based switched methodology for the identification of a perturbed sinusoidal signal: Theory and experiments," IEEE Trans on Signal Processing, vol. 62, no. 24, pp. 6355-6365, Dec 2014.

[17] B. Chen, G. Pin, W. M. Ng, S. Y. R. Hui, and T. Parisini, "An adaptive-observer-based robust estimator of multi-sinusoidal signals," IEEE Trans on Automatic Control, vol. 63, no. 6, pp. 1618-1631, June 2018.

[18] G. Pin, Y. Wang, B. Chen, and T. Parisini, "Identification of multi- sinusoidal signals with direct frequency estimation: An adaptive observer approach," Automatica, vol. 99, pp. 338 - 345, 2019.

[19] B. Chen, P. Li, G. Pin, G. Fedele, and T. Parisini, "Finite-time estimation of multiple exponentially-damped sinusoidal signals: A kernel-based approach," Automatica, vol. 106, pp. $1-7, \quad 2019$. [Online]. Available: http://www.sciencedirect.com/science/article/pii/S0005109819301761

[20] G. Pin, B. Chen, T. Parisini, and M. Bodson, "Robust sinusoid identification with structured and unstructured measurement uncertainties," IEEE Trans on Automatic Control, vol. 59, no. 6, pp. 1588-1593, June 2014.

[21] B. Chen, G. Pin, W. M. Ng, S. Y. R. Hui, and T. Parisini, "A parallel prefiltering approach for the identification of a biased sinusoidal signal: Theory and experiments," Int. J. Adaptive Control and Signal Proc., vol. 29, no. 12, pp. 1591-1608, 2015.

[22] G. Pin, B. Chen, and T. Parisini, "Robust finite-time estimation of biased sinusoidal signals: A volterra operators approach," Automatica, vol. 77 , pp. 120 - 132, 2017.

[23] L. Dieci and L. Lopez, "Sliding motion in filippov differential systems: Theoretical results and a computational approach," SIAM J. on Numerical Analysis, vol. 47, no. 3, pp. 2023-2051, 2009.

[24] I. Barkana, "Defending the beauty of the invariance principle," Int $J$. Control, vol. 87, no. 1, pp. 186-206, 2014.

[25] — - "Barbalats lemma and stability - misuse of a correct mathematical result?" Mathematics in Engineering, Science and Aerospace (MESA), vol. 7, no. 1, pp. 197-218, 2016.

[26] M. Hou, "Parameter identification of sinusoids," IEEE Trans on Automatic Control, vol. 57, no. 2, pp. 467-472, 2012 